\title{
EchoGéo
}

$4 \mid 2008$

Archéologie et Géographie

\section{Le temps des géographes}

Jean Marie Théodat

\section{(2) OpenEdition}

Journals

Édition électronique

URL : https://journals.openedition.org/echogeo/2404

DOI : $10.4000 /$ echogeo.2404

ISSN : 1963-1197

\section{Éditeur}

Pôle de recherche pour l'organisation et la diffusion de l'information géographique (CNRS UMR 8586)

Référence électronique

Jean Marie Théodat, «Le temps des géographes », EchoGéo [En ligne], 4 | 2008, mis en ligne le 25 janvier 2010, consulté le 01 août 2021. URL : http://journals.openedition.org/echogeo/2404 ; DOI : https://doi.org/10.4000/echogeo.2404

Ce document a été généré automatiquement le 1 août 2021

EchoGéo est mis à disposition selon les termes de la licence Creative Commons Attribution - Pas d'Utilisation Commerciale - Pas de Modification 4.0 International (CC BY-NC-ND) 


\title{
Le temps des géographes
}

\author{
Jean Marie Théodat
}

1 Dans les représentations du temps, la tendance commune est de considérer l'avenir comme un horizon lointain ouvert devant nos pas, le passé comme un monde insondable fermé dans notre dos, et le présent comme une lande offerte où il nous suffirait d'enchaîner les pas pour avancer. Les commémorations et la pompe coutumière des cérémonies officielles ponctuent cette marche linéaire de dates mémorables que les générations ont conservées comme des balises mentales dans le continuum de la vie. Les occasions ne manquent pas où la pensée commune interroge l'événement au travers de prismes hérités d'une représentation ancienne du monde tandis que la réalité ne cesse d'évoluer, de soumettre le réel à de nouveaux défis. Et pourtant, comprendre ces nouveaux défis suppose un ajustement permanent de la méthode et des outils, de recourir à de nouveaux paradigmes. Ces approches nouvelles dans l'évaluation de l'impact des événements sur l'organisation des affaires humaines à différents pas d'échelles spatiale et de durée donnent l'impression d'un émiettement de la discipline, mais cette division spécialisée de la tâche est la condition de l'efficacité de l'éclairage commun. C'est à cet exercice qu'invite la dernière livraison de la revue Echogéo.

2 Qu'il s'agisse des phénomènes physiques relevant de l'investigation scientifique la plus objective ou des événements politiques récents qui ponctuent d'alertes sanglantes l'actualité (donc la subjectivité) internationale, la revue a donné la parole à ceux dont le métier est d'éclairer et de nous rendre intelligible l'actualité à travers l'éclairage d'un passé plus ancien.

3 Toujours avec le souci de comprendre ce qui s'est passé et d'assigner à des principes rationnels les grandes mutations à l'œuvre dans le monde, nous avons limité le champ de notre intérêt, dans ce numéro, à des questions s'inscrivant dans des terrains variés et qui attestent de l'importance de la durée et de l'approche diachronique dans l'analyse géographique.

4 Parce que la compréhension du monde suppose le recours à des mesures de plus en plus fines requérant des instruments de plus en plus sophistiqués, la discipline géographique se complexifie et propose des champs d'investigation empruntant à des 
traditions scientifiques annexes telles que l'archéologie et l'anthropologie pour donner naissance à deux courants, correspondant à des méthodologies distinctes de la géographie : la géoarchéologie et archéogéographie.

Dans la rubrique Sur le Champ ces deux approches complémentaires sont déclinées sous la forme d'une analyse en trois volets correspondant à trois pas d'échelle, et qui mettent successivement en exergue les ressources des récits anciens confrontés aux méthodes et aux résultats des fouilles archéologiques pour parvenir à une intelligence des modes d'appropriation symbolique du territoire, avec Dominique Guillaud (Sumatra) ; puis il est question, avec Corinne Feiss, J.M. Mouton, C. Piaton, P.J. Jehel, et Jeaninne le Rhun, de la part des données météorologiques et tectoniques anciennes dans la détermination de la situation d'une forteresse médiévale à Sadr (Sinaï); enfin Gilles Arnaud-Fassetta démontre l'intérêt de l'analyse paléohydrographique dans la compréhension des anciens réseaux de drainage. Dans cette dernière approche le recours à des mesures hydrographiques et hydrologiques accorde une moindre place à la mémoire, au discours, dans la compréhension des faits, en l'occurrence les migrations de l'habitat dans les vallées où les sociétés ont eu à affronter le risque lié à l'aléa hydrologique, mais c'est toujours le passé ancien qui perce à travers les grilles de l'analyse.

6 Dans la rubrique Sur le Métier, Etienne Cossart et Julie Le Gall abordent la question des conséquences hydrologiques et humaines du recul des glaciers du massif de l'Aconcagua (Argentine) en recourant à une évaluation sur la longue durée du réchauffement subi depuis le Petit Age glaciaire. De même dans la rubrique Sur le Terrain, Marie Chenet et Erwan Roussel évaluent les nouvelles dynamiques induites sur les versants libérés du gel par le recul généralisé des glaciers dans le cas du Morsàrjökull et du Skaftafellsjökull (Islande). Une continuité thématique oblique nous conduit à l'ouvrage de Stéphane Ghiotti, Les territoires de l'eau, dont Olivier Ninot présente les grandes lignes et souligne l'intérêt dans la compréhension de sa gestion en rapport avec la thématique du développement en France.

7 La rubrique Sur le Vif, dont l'alimentation à flux continu le distingue du reste de la publication, revient sur un certain nombre de faits qui, pour relever de l'actualité la plus brûlante, n'en participent pas moins d'une temporalité plus longue qui nous oblige à nous pencher sur les motifs constants, intemporels, qui en sont la clé d'intelligence. Vous lirez avec intérêt les analyses sur: la question de l'adhésion de la Turquie à l'Union Européenne (Guy Baudelle), l'évolution de la situation en Afghanistan (Nathalène Reynolds), la place de la question kurde dans le règlement global de la crise au Moyen-Orient (Saywan Barzani), l'ombre portée du mur sur la complexité de la crise entre Israël et la Palestine (Pierre Renno), l'interprétation de la visite d'Etat du colonel Kadhafi à Paris dans la perspective des relations entre l'Union Européenne et la rive Sud de la Méditerranée (Pierre Beckouche), les réactions à Belgrade de la proclamation de l'indépendance du Kosovo (Amaël Cattaruzza), les leçons de la guerre civile au Tchad (Géraud Magrin) et au Kenya (Claire Médard).

8 Entre le passé ancien des glaciers et l'actualité récente, si brûlante qu'elle semble porter en elle l'avenir, ce cahier, par les contributions qu'il rassemble, montre l'articulation entre des temporalités et des échelles différentes pour la compréhension du réel. Le temps est ici conçu non plus à la manière d'un continuum allant du passé au futur, mais comme un champ de forces où s'affrontent des contraires. Hannah Arendt imagine le présent comme un intervalle où s'inscrit la conscience. Alors, tandis qu'un 
passé ancien refoule son élan, un avenir pressant le pousse à l'engagement, faisant de son présent une brèche ardente où la condition humaine se réduit à une lutte sans pause pour rester tout simplement debout dans l'action. Dans cette perspective, le métier de penser est un sursaut au-dessus de la brèche, « un petit tracé de non-temps que l'activité de la pensée inscrit à l'intérieur de l'espace-temps des mortels et dans lequel le cours des pensées sauve tout ce qu'il touche de la ruine du temps historique» (La crise de la culture, 1954). 